\title{
De la fenêtre du train en mouvement
}

Tommaso Meldolesi

\section{OpenEdition}

\section{Journals}

Édition électronique

URL : https://journals.openedition.org/rhcf/2007

DOI : 10.4000/rhcf.2007

Éditeur

Rails \& histoire

Édition imprimée

Date de publication : 1 février 2003

Pagination : 250-261

ISBN : 0996-9403

ISSN : 0996-9403

Référence électronique

Tommaso Meldolesi, « De la fenêtre du train en mouvement », Revue d'histoire des chemins de fer [En ligne], 26 | 2003, mis en ligne le 22 janvier 2015, consulté le 22 avril 2022. URL : http:// journals.openedition.org/rhcf/2007 ; DOI : https://doi.org/10.4000/rhcf.2007 


\section{De la fenêtre du train en mouvement}

Au cours de la période comprise entre le commencement de l'ère du rail et le tout début du $\mathrm{xx}^{\mathrm{e}}$ siècle, l'accélération de la vitesse des trains produit chez les voyageurs de l'époque à la fois une confusion et une prolifération d'images, relevant des visions depuis la fenêtre d'un convoi en mouvement aussi bien que de l'imagination et des troubles du passager en chemin de fer.

Le paysage en mouvement, perçu par l'œil humain à une vitesse infiniment supérieure qu'elle ne l'avait été pour les passagers habitués aux allures régulières des diligences, des carrosses ou des fiacres, constitue un élément de trouble pour le voyageur qui regarde. Celui-ci a parfois l'impression d'être transporté dans un autre monde géré par des forces magiques qui à la fois l'attirent et l'effraient énormément.

Les paysages, en effet, bien nets sous les yeux des voyageurs en diligence qui pouvaient en saisir le moindre détail, sont remplacés par des raies, de couleur différentes, qui passent, le temps d'un éclair, sous les yeux ébahis et troublés du voyageur en chemin de fer.

Dans la mesure où il n'a plus la possibilité de contrôler de manière plus ou moins directe le moyen de locomotion qui le transporte, l'individu se trouve dans une situation d'abandon au mouvement du convoi ; son regard se penche du côté de la fenêtre dans la contemplation du paysage. Cependant, l'image du paysage est généralement limitée. Elle n'atteint pas des distances infinies et correspond essentiellement à l'ampleur du champ visuel propre au voyageur. Cela engendre l'idée d'« espace du paysage » qui se transforme en « espace géographique ».

Dès l'introduction des chemins de fer, les espaces géographiques ne correspondent plus à des paysages bien déterminés mais changent continuellement, grâce à la vitesse du convoi. Les paysages se transforment en petits points et en lignes ou en courbes changeantes ne correspondant plus aux formes et aux silhouettes que les hommes avaient vues pendant des siècles. Les espaces sont dévorés à des vitesses impensables jusque-là. Quelques décennies avant la naissance du cinéma ${ }^{1}$,

1- « La visione attraverso la porta di una carrozza ferroviaria in movimento, o attraverso il parabrezza di un'automobile è frammentata, sebbene alle alte velocità essa diventi continua, nel modo in cui nel cinema la continuità è creata da una serie di fotografie in posa. » (La vision à travers la portière de la voiture ferroviaire en mouvement ou à travers le pare-brise d'une voiture est fragmentée, bien qu'aux grandes vitesses elle devienne continue, par la manière dans laquelle au (suite page suivante) 
les yeux ébahis des voyageurs ferroviaires s'étonnent devant des arbres et des maisons qui « dansent » frénétiquement au-delà de la fenêtre du convoi en mouvement, et se volatilisent. La vitesse arrache au regard les objets et suscite en même temps un accroissement du nombre des impressions et des images que l'œil voit défiler. L'espace que le voyageur est en train de parcourir se transforme en tableaux, en images à travers une accumulation et un échange très rapide et continu entre les impressions intérieures et extérieures qui finissent par accabler l'esprit et la conscience du passager. Malgré les efforts inutiles et la fatigue que le voyageur emploie pour essayer de les retenir, il ne réussira pas dans ses intentions et se servira de son imagination afin de recomposer la réalité fragmentée qui apparait devant ses yeux. Alors, afin de saisir le plus possible l'ensemble du paysage, les voyageurs se penchent de tous les côtés :

$\mathrm{A}$ un moment où je dénombrais les pensées qui avaient rempli mon esprit pendant les minutes précédentes, pour me rendre compte si je venais ou non de dormir (et où l'incertitude même qui me faisait me poser la question était en train de me fournir une réponse affirmative) dans le carreau de la fenêtre au-dessus d'un petit bois noir, je vis des visages échancrés dont le doux duvet était d'un rose fixé, mort, qui ne changera plus, comme celui qui teint les plumes de l'aile qui l'a assimilé ou le pastel sur lequel l'a déposé la fantaisie du peintre. Mais je sentais qu'au contraire cette couleur n'était ni inertie, ni caprice, mais nécessité et vie. Bientôt s'amoncelèrent derrière elle des réserves de lumière. Elle s'aviva. Le ciel devint d'un incarnat que je tâchais, en collant mes yeux à la vitre, de mieux voir car je le sentais en rapport avec l'existence profonde de la nature, mais la ligne du chemin de fer ayant changé de direction, le train tourna, la scène matinale fut remplacée dans le cadre de la fenêtre par un village nocturne aux toits bleus de clair de lune, avec un lavoir encrassé de la nacre opaline de la nuit, sous un ciel encore semé de toutes ses étoiles, et je me désolais d'avoir perdu ma bande de ciel rose quand je l'aperçus de nouveau, mais rouge cette fois, dans la fenêtre d'en face qu'elle abandonna à un deuxième coude de la voie ferrée ; si bien que je passais mon temps à courir d'une fenêtre à l'autre

(suite) cinéma la continuité est créée par une série de photographies en pose.) (notre traduction), Stephen Kern, The Culture of Space and Time (1880-1918), Cambridge (USA), Harvard Massachussets University Press, 1983 (trad. ital. par Barnaba Maj : Il Tempo e lo spazio, la percezione del mondo tra Otto e Novecento, Bologna, Il Mulino, 1988, p. 151). 
pour rapprocher, pour rentoiler les fragments intermittents et opposites de mon beau matin écarlate et versatile et en avoir une vue totale et un tableau continu ${ }^{2}$.

Ailleurs, en revanche, bien qu'il existe la possibilité d'observer plus d'un paysage, certains voyageurs n'ont pas l'air de s'en apercevoir : c'est le cas chez Jules Verne. Un passage, tiré de Michel Strogoff (1876) et concernant le voyage de Moscou à Nijni-Novgorod, décrit ainsi le paysage :

Et, de plus, comme Harry Blount, assis à la gauche du train, n'avait vu qu'une partie de la contrée, qui était assez accidentée, sans se douter à peine de regarder la partie de droite formée de longues plaines, il ne manqua pas d'ajouter, avec l'aplomb britannique :

«Pays montagneux entre Moscou et Wladimir $»^{3}$.

Le procédé utilisé par Verne pour décrire la variation du paysage est tout à fait novateur pour son époque. Verne introduit deux fenêtres (une de chaque côté) par lesquelles il est possible de voir deux paysages différents et même opposés. Cette technique révolutionne la perception du paysage et met en relief non seulement sa mobilité mais également sa relativité. Le voyageur voit ce qu'il veut observer, mais sa perception des images sera toujours partielle et incomplète, simplement parce qu'elle cachera à son regard l'autre côté des choses.

On remet en question l'idée de panorama qui ne correspond plus à partir de ce moment à un tableau fixe, immobile et inaltérable. Bien au contraire, l'image de " panorama » évoquée dans l'étude de Wolfgang Schivelbusch ${ }^{4}$ prend une importance capitale à travers les yeux du voyageur : le chemin de fer forge lui-même le paysage que l'on perçoit en mouvement à travers la fenêtre du convoi.

Tout cela provoque des sentiments de trouble chez le voyageur qui ressent l'impression, comme c'était le cas de John Ruskin, d'être transporté passivement à la manière d'un paquet et de perdre tout plaisir dans l'observation du paysage dans la mesure où il ne parvient pas à en apprécier les sons et les parfums.

2- Marcel Proust, $A$ l'ombre des jeunes filles en fleur (1919), A la Recherche du temps perdu, éd. Jean-Yves Tadié, Paris, Bibliothèque de La Pléiade, 1988, p. 15-16.

3- Jules Verne, Michel Strogoff (1876), Paris, Le livre de poche, ${ }^{\circ} 2034,1994$, p. 50.

4- Wolfgang Schivelbusch, «Die panoramische Reise », Geschichte der Eisenbahnreise, Carl Hanser Verlag, Munich, 1977 («Le voyage panoramique », Histoire des voyages en train, Paris, Le Promeneur, 1990, p. 57-75). 
Image donc incomplète dans sa totalité. Image fugace, image instable, insaisissable, étrange, parfois presque imperceptible. Le monde réel devient, aux yeux du voyageur ferroviaire, comme une suite de tableaux prenant forme à une distance variable et que le passager n'aperçoit qu'à travers la glace de la fenêtre qui l'en sépare. Se trouve-t-il alors dans une situation de rêve, de fantasmagorie ou est-il encore bien ancré et présent dans la réalité dont il n'est plus entouré, mais qu'il traverse à toute allure à bord d'un monstre dévorant les espaces à une vitesse et dans des temps qui, au début de l'ère du chemin de fer, n'avaient pas d'équivalents ?

L'image qui fuit s'enracine pourtant dans l'esprit du voyageur. Celui qui fait un voyage en chemin de fer et parcourt plusieurs fois le même trajet trouve devant ses yeux maints paysages caractérisés par une infinité d'objets et d'éléments nouveaux. A chacun de ces aspects le voyageur donnera une signification particulière qui dépendra de son état d'âme du moment ou de la première impression qu'un objet, une image, un élément du paysage auront suscitée en son âme. C'est l'image fugace qui contient en elle le sens même de son voyage, de sa curiosité, de sa recherche. C'est elle qui détermine l'élan du voyageur. Sa volatilisation la rend insaisissable, idéalisée, absolue.

Il peut alors arriver que, comme l'affirme Schivelbusch, « La volatilisation de la réalité et sa résurrection comme panorama constituent la condition pour que la vue s'émancipe totalement du paysage traversé et se plonge dans un pays de substitution imaginaire, la littérature $»^{5}$. Tout cela est accompli par un voyageur curieux, en quête de nouveautés, qui exprime ses sensations à travers la production littéraire.

Deux textes méconnus servent d'introduction à la « littérature de l'image ferroviaire ». Le premier date de 1835. Son auteur, une femme d'origine belge, Mme Félix de la Motte, résume ses impressions de voyage dans l'un des poèmes tirés de son recueil Les Violettes ${ }^{6}$ :

Quand la Flèche, ardente fournaise,

Vomissait la fumée et répandait sa braise

Quand les champs tournoyants fuyaient devant mes yeux...

5- Ibid., p. 69.

6- Le poème fut composé en juin 1835 au lendemain de l'inauguration de la ligne Malines-Bruxelles (mai 1835), mais il n'est publié que l'année suivante avec l'ensemble du recueil.

7-Texte cité par Claude Pichois, Littérature et progrès, vitesse et vision du monde, Neuchâtel, La Baconnière, coll. «Langages », 1973, p. 24. 
Le second document important pour la littérature cinétique est un texte en prose, composé cette fois-ci par un représentant du courant saint-simonien $^{8}$, Charles-Claude Ruelle, connu sous le pseudonyme de Claudius. Il fut écrit à l'occasion de l'inauguration de la voie ferrée reliant Paris à Saint-Germain en-Laye en août 1837 et avait un but didactique : montrer à tous ceux qui ne pouvaient pas se permettre de voyager en train quelles étaient les sensations que l'on éprouvait au cours du voyage.

[...] puis, sous la lueur de cette fournaise qui marche, quel travail précipité ! quelle agitation désordonnée ! quel combat ! nulle vibration de bras qui puisse donner une idée de ce terrible va et vient, de cet entraînement frénétique ; et derrière, qui rendra l'effet écrasant de ces grandes masses sombres que l'œil ose à peine compter, que l'imagination la plus hardie n'ose à peine soulever, et qui pourtant glissent au-dessus de votre tête comme un oiseau ? Devant ce tournoiement qui n'a rien d'humain, devant cette rangée de maisons qui fuient au pas de course, vous restez anéanti. D'un peu plus loin, vous eussiez dit je ne sais quel corps étranger à la terre, je ne sais quelle planète égarée. Ce nocturne spectacle semble fait pour nous imiter à ce furieux mouvement dont les chiffres astronomiques nous parlent avec tant de froideur; renouvelé chaque soir, il est à penser qu'il aura bientôt perdu ce qu'il a de bizarre.

[...] il faut monter ce coursier d'invention humaine ; il faut traverser à sa suite souterrains, arceaux de pont, villages, plaines, bois, rivière ; il faut voir fuir, près de soi, la poussière du chemin comme un courant torrentueux, ou bien comme une toile ondoyante soulevée par le vent; il faut voir les ouvriers de la route passer, emportés de toute pièce dans leur immobilité, comme des figures de carton sur une décoration mouvante?

8- Interprète d'un courant pacifiste prêchant la communion et la fraternité des peuples, remontant à Charles-Henri de Saint-Simon disparu en 1825, trop tôt pour se rendre compte de ce qu'était vraiment le chemin de fer. Ses disciples, cependant, et parmi eux Prosper Enfantin, pensaient déjà autour de 1830 que les nouveaux et puissants moyens de communication auraient poussé les masses populaires vers un nouvel intérêt commun pour le travail et vers un nouveau sens esthétique. «Réagissant contre l'esthétique individualiste [ce mouvement] assignait à l'art la mission de donner aux masses une âme commune et d'émouvoir un peuple laborieux dans l'intérêt même du travail. » Le Producteur I, Journal philosophique, de lindustrie, des sciences et des beaux-arts, 1825, p. 176-177. (Texte cité par Marc Baroli, Le Train dans la littérature française, Paris, Éditions NM, 1969, p. 21.)

9- Charles-Claude Ruelle, La Science populaire de Claudius. Simples discours sur toutes choses. Chemins de fer et voitures à vapeur (1835). (Texte cité par Claude Pichois, op. cit., p. 22-23.) 
C'est là un texte capital concernant la littérature de chemin de fer, qui d'une part cherche à faire connaitre l'inconnu et fait face courageusement aux énigmes que la technique et l'industrie posent à la culture et à la vie des gens ; et d'autre part examine la position de l'observateur qui se croit immobile tout en bougeant à cause du mouvement du convoi. Il s'agit donc d'une sorte de paradoxe que l'auteur expose à un vaste public dans l'espoir de faire connaitre rapidement le nouveau moyen de locomotion.

C'est à la suite d'un voyage en chemin de fer, de Paris à Bruxelles, en 1837, que Victor Hugo s'exprime ainsi :

C'est un mouvement magnifique et qu'il faut avoir senti pour s'en rendre compte. La rapidité est inouie. Les fleurs du bord du chemin ne sont plus des fleurs, ce sont des taches ou plutôt des raies rouges et blanches ; plus de points, tout devient raie ; les blés sont de grandes chevelures jaunes, les luzernes sont de grandes chevelures vertes ; les villes, les clochers et les arbres dansent et se mêlent follement à l'horizon; de temps en temps, une ombre, une forme, un spectre parait et disparait comme l'éclair à côté de la portière ; c'est une garde du chemin qui, selon l'usage, porte militairement les armes du convoi. On se dit dans la voiture : c'est à trois lieues, nous y serons dans dix minutes ${ }^{10}$.

A partir de ce texte nous pouvons constater la présence d'éléments évoquant la déformation du paysage. Par la «vitesse inouie », en effet, les divers éléments du paysage sont transformés en taches. Les « chevelures » jaunes et vertes évoquent à l'esprit les images des futurs tableaux impressionnistes de Vincent Van Gogh ${ }^{11}$ flottant dans l'air grâce au " mouvement magnifique qu'il faut avoir ressenti pour s'en rendre compte ».

Un pareil enthousiasme envers le voyage ferroviaire est perceptible dans un feuilleton La Vie en chemin de ferécrit par Benjamin Gastineau et publié en 1861. Cet auteur est un grand partisan du chemin de fer. Il écrit dans son œuvre :

A travers les carreaux chargés de vapeur du wagon on croit voir passer spectres et fantômes. Les tombes du cimetière surgissent, sépulcres blanchis, au sein de la nuit noire; une lumière isolée scintille comme l'œil de Satan. Les morts se lèvent de leurs tombes, se livrent à un effroyable steeple-chase avec le chemin de fer qui fuit plus rapide que le cheval macabre de Lénore. Sur son

10- Victor Hugo, Vyage en Belgique (1837), Voyages, Paris, Hachette, 1950, p. 32. 11- Tel par exemple Le Champ de blé à l'alouette de 1887. 
passage les grands bois murmurent des choses sinistres : ils s'entretiennent des crimes des humains ou se moquent de leurs innombrables bévues. Le voyageur que ne rassure certes pas la lampe funéraire de son wagon sent une sueur glaciale coller sa chemise à ses os. Heureusement, bandes de ténèbres, légions de spectres, s'enfuient devant le disque d'or de la lune qui se montre au fond de l'horizon et devant la lumière des étoiles blondes, s'allumant l'une après l'autre au ciel ${ }^{12}$.

L'auteur propose des descriptions quasi fantastiques, en même temps qu'il affirme le rôle important et formateur du chemin de fer dans la société aussi bien qu'au sein de la nature :

Avant la création du chemin de fer, la nature ne palpitait plus ; c'était une Belle-au-bois-dormant, une froide statue, un végétal, un polype ; les cieux mêmes paraissaient immuables. Le chemin de fer a tout animé, tout mobilisé... Le ciel est devenu un infini angoissant, la nature une beauté en action. Le Christ s'est détaché de sa croix, il a marché et il a laissé bien derrière lui, sur la route, le vieil Ahasvérus. Semblable à une capricieuse femme jonglant avec le cœur de son amant, le wagon joue avec le ciel, le fleuve, le nuage, les sites riants et sombres, tantôt vous montrent la vie en noir, tantôt en rose, tantôt vous transportent sur la cime d'une montagne, tantôt vous plongent dans un abîme... ${ }^{13}$

L'enthousiasme pousse donc Gastineau à affirmer que le train s'avère utile non seulement pour la société, mais également pour la nature qui a l'impression de renaitre sous l'influence de la nouvelle force motrice du chemin de fer. La fonction ludique du wagon, presque naif, jouant avec les éléments de la nature et transportant le voyageur, tantôt au sommet d'une montagne, tantôt « dans l'abîme », en lui montrant la beauté et la laideur de la vie, est accentuée dans la suite du passage :

Le chemin de fer prête les charmes du mouvement et de l'émotion au paysage qu'il voile ou dévoile, qu'il fait rire ou pleurer, qu'il estompe, dessine ou peint par grands plans. Par l'action rapide du convoi tout se transforme, se profile, se poétise. Les dieux morts ressuscitent, les grossiers Marions s'idéalisent, la pierre fait statue, l'arbre pérore ou pleure, le nuage ivre va du zénith au nadir; les forêtes se prennent aux cheveux, font une mêlée furieuse, le soleil danse le menuet, et la lune s'égare... ${ }^{14}$

12- Benjamin Gastineau, La Vie en chemin de fer (1861), Paris, Dentu, 1861, p. 53-54. 13- Ibid., p. 48-50.

14- Ibid. 
A travers cette dernière description revient chez Gastineau l'idée du paysage en mouvement que nous avons déjà analysée dans le Voyage en Belgique de Victor Hugo. Par rapport à ce dernier, La Vie en chemin de fer met en place un ensemble d'images riches en métaphores où le paysage revient à la vie grâce à l'action créatrice du convoi.

Plus réaliste que Gastineau, Jules Clarétie, journaliste et publiciste parisien d'origine limousine, dans Voyages d'un Parisien, en 1865, se sert dans sa description du concept de panorama afin de caractériser la fuite des objets et des images permettant de tout saisir et de tout embrasser dans un même regard :

En quelques heures, il vous présente toute une France, sous vos yeux se déroule la totalité du panorama, une succession rapide d'images charmantes et de surprises toujours nouvelles. Il vous montre purement et simplement l'essentiel d'une région, en vérité c'est un artiste dans le style des anciens maitres. N'exigez de lui aucun détail mais seulement le tout où est la vie. Enfin, après qu'il vous a ravi par la fugue du coloriste, il s'arrête et vous libère à votre but ${ }^{15}$.

La plupart des textes cités est constituée de «mosaïques d'images » qui se lient, s'enchaînent et se juxtaposent pour former des paysages en mouvement. Aux yeux du voyageur voulant s'emparer d'un seul regard du paysage dans sa totalité, les images, aperçues du convoi en mouvement, prennent à la fois des traits irréels et magiques.

La fantaisie, stimulée par ces mêmes images, se déclenche dans une course effrénée en compétition avec le chemin de fer. C'est ce qu'on trouve dans les vers du fondateur du mouvement futuriste italien, Filippo Tommaso Marinetti, qui écrivait en langue française, au tout début du $\mathrm{xx}^{\mathrm{e}}$ siècle :

Mon train enragé d'éclairs verts et de vents

Fuit sans cesse en roulant son galop de tonnerre,

Avec des bonds, des soubresauts, des demi-tours

Et d'élégantes vire-voltes sur la courbe des rails

Qui luisent en plongeant dans le noir,

Avec un espagnol et dangereux déhanchement

A pic sur les abîmes insondables...

$[\ldots]$

15- Jules Clarétie, Voyages d'un Parisien, Paris, 1865, p. 4 (Texte cité par W. Schivelbusch, op. cit., p. 66-67.) 
Montagnes, ô bétail monstrueux, ô mammouths

Qui trottez lourdement, arquant vos dos immenses, Vous voilà dépassés, laissés bien loin, noyés.

$[\ldots]$

Hourrah! hourrah! les vents qui me gaffez

D’un coup de gaule au tournant des vallées

Je vous ai dépassés, émiettés et vaincus !...

Hourrah! hourrah! Vaincus ou dépassées

Les villes mornes crucifiées,

Sur les grands bras en croix des routes blanches $! . .{ }^{16}$.

Cela correspond à une mutation de perspective dans la perception des images qui est déclarée, du point de vue théorique, par un autre représentant du Futurisme italien :

Nous pouvons étudier - c'est à dire aimer - une machine, n'importe quelle rotative et nous servir de ses plans, de ses profils, de ses creux, de ses mouvements tout comme d'éléments naturels pour la construction de notre paysage. Des arbres et des branches, ne sont-ils pas peut-être les parties d'un mécanisme primordial ? Tout est beauté naturelle, non pas pour l'apparence extérieure, mais pour ses significations plastiques abstraites ${ }^{17}$.

Cette nouvelle perception du paysage comme un flux ininterrompu et violent d'images est présent au-delà des Futuristes ; dans la Lettre d'un ami dans Monsieur Teste Valéry présente une scène où :

[...] Le train filait toujours, rejetant violemment peupliers, vaches, hangars, et toutes choses terrestres, comme s'il avait soif, comme s'il courait à la pensée pure, ou vers quelque étoile à rejoindre. Quel but suprême peut exiger un ravissement si brutal, un renvoi si vif de paysages à tous les diables ${ }^{18}$ ?

16- Filippo Tommaso Marinetti, Le Démon de la vitesse V (1902), Destruction, Poèmes lyriques, Paris, Vanier, 1904, p. 101, v. 79-85 ; p. 126, v. 163-165 et 172-177. 17- Umberto Boccioni, «Contro il paesaggio e la vecchia estetica», Pittura, scultura futuriste, Milano, Edizioni futuriste di « Poesia », 1914. Texte cité par Luciano De Maria, Filippo Tommaso Marinetti e il Futurismo, Milano, Mondadori, Oscar, 1973, p. 214 (notre traduction).

Cette nouvelle perception et transformation de la réalité et du paysage à travers la juxtaposition des plans d'une machine sera par ailleurs en Italie à la base des Quaderni di Serafino Gubbio operatore, le roman que Pirandello publie en 1915.

18- Paul Valéry, Lettre d'un ami, Monsieur Teste, Paris (1896), Gallimard, coll. «L'Imaginaire », 1999, p. 85. 
Les sensations relatives à un mouvement impossible à réfréner et inquiétant sont ailleurs également présentes dans Lucienne de Jules Romains :

Tous les rails comme des crins dorés fuyaient devant nous, se serraient peu à peu en touffe et montaient en même temps vers un point du ciel noir où commençaient les étoiles. Ces fils d'or étaient si parfaitement tendus, ils allaient se rejoindre d'un mouvement si beau, que les yeux ne semblaient pas suffire pour en comprendre l'harmonie ${ }^{19}$.

À la différence des textes précédents, Lucienne reste immobile sur le ballast de la gare. Les images évoquées sont une projection de ses pensées. L'image des « fils dorés » fuyant et se serrant « en touffe » reprend d'une part l'idée de la rapidité de la perception du paysage que l'état d'âme de Lucienne arrive difficilement à maitriser ; d'autre part, l'opposition entre l'or des fils et le " point noir du ciel » est à l'origine d'un état évident d'inquiétude chez la protagoniste dont l'âme est troublée par la fuite incessante et continue des divers éléments du paysage.

Nous retrouvons, dans les derniers textes cités, une participation des mouvements de l'âme et de la conscience qui interviennent en se superposant aux images du paysage dont les caractéristiques physiques sont presque inexistantes. Le passager se trouve tout seul dans un compartiment. Il a l'impression d'être loin du monde et ses yeux sont de moins en moins intéressés par le défilé des paysages qui lui passent sous les yeux. À cause de l'accumulation d'images entrevues par la fenêtre du convoi la foule désordonnée des souvenirs s'amoncelle dans l'âme du voyageur, causant son mutisme, son blocage et, enfin, son incapacité à voir le paysage extérieur, se contentant d'entrevoir une sorte de paysage intérieur qu'il construit en lui lors du voyage en train. La conséquence des réflexions individuelles face au paysage qui défile sans arrêt autour de lui mène le voyageur à une situation de mutisme et d'aveuglement qui l'isole complètement de ce qui se passe en dehors de lui. Parfois, comme dans le dernier exemple cité, cela correspond non pas à des visions véritables mais à une projection des rêves d'un personnage dans un espace fictif, loin du réel. La vitesse des images ne concerne alors plus simplement le chemin de fer mais les rythmes obsédants de toute une société qui est en train de se transformer. L'individu s'isole et s'éloigne du monde, renfermé de plus en plus dans ses rêves, ses visions, ses fantaisies, ses hantises, ses fantasmes dus aux regards trop rapides qui s'associent mal au rythme de son existence. Le voyageur aurait besoin de davantage de tranquillité, de davantage de temps et de moins de

19- Jules Romains, Lucienne (1922), Paris, Le livre de poche, 1964, p. 26-27. 
contraintes de la part d'une société fondée sur l'idée de vitesse, sur la conquête de l'espace, sur la perception instantanée des images et des choses. Il s'agit d'une sorte d'épreuve à laquelle l'homme est nécessairement soumis sous peine de ne pas pouvoir s'intégrer, ni de collaborer ou de vivre dans un monde de plus en plus rapide.

\section{Bibliographie générale}

- Bachelard, Gaston, La Poétique de l'espace, Paris, Puf, coll. "Quadrige », 1998.

- Baroli, Marc, Le Train dans la littérature française, Paris, Éditions NM, 1969.

- Boccioni, Umberto, «Contro il paesaggio e la vecchia estetica », Edizioni futuriste di « Poesia », 1914, Luciano de Maria, Filippo Tommaso Martinetti e il Futurismo, Milano, Mondadori, Oscar, 1973.

- Chevalier, Jean ; Gheebrant, Alain, Dictionnaire des Symboles, Paris, Laffont, coll. «Bouquins », 1982.

- Clarétie, Jules, Voyages d'un Parisien, Paris, 1865.

- Deleuze, Gilles, Cinéma. L'image-Mouvement, Paris, Les éditions de Minuit, 1983.

- De Maria, Luciano, Filippo Tommaso Marinetti e il Futurismo, Milano, Mondadori, Oscar, 1973.

- Gastineau, Benjamin, La Vie en chemin de fer (1861), Paris, Dentu, 1861.

- Hugo, Victor, Voyage en Belgique (1837), Voyages, Paris, Hachette, 1950.

- Kern, Stephen, The Culture of Space and Time (1880-1918), Cambridge (USA), Harvard Massachussets University Press, 1983 (trad. ital. par Barnaba Maj : Il Tempo e lo spario, la percerione del mondo tra Otto e Novecento, Bologna, Il Mulino, 1988).

- Le Producteur I, Journal philosophique, de lindustrie, des sciences et des beaux-arts, 1825.

- Marinetti, Filippo Tommaso, Le Démon de la vitesse V (1902), Destruction, Poèmes lyriques, Paris, Vanier, 1904.

- Pichois, Claude, Littérature et Progrès, vitesse et vision du monde, Neuchâtel, La Baconnière, coll. « Langages », 1973. 
- Pirandello, Luigi, Quaderni di Serafino Gubbio operatore (1915), Tutti i Romanzi, t. 2, éd. Giovanni Macchia, Milan, Mondatori, coll. "I Meridiani », 1990, p. 517-735.

- Proust, Marcel, A l'ombre des jeunes filles en fleur (1919), A la Recherche du temps perdu, éd. Jean- Yves Tadié, Paris, Bibliothèque de La Pléiade, 1988.

- Romains, Jules, Lucienne (1922), Paris, Le livre de poche, 1964.

- Ruelle, Charles-Claude, La Science populaire de Claudius. Simples discours sur toutes choses. Chemins de fer et voitures à vapeur (1835).

- Schivelbusch Wolfgang, Geschichte der Eisenbahnreise, Munich, Carl Hanser Verlag, 1977 (trad. française par J.-F. Boutout, Histoire des voyages en train, Paris, Le Promeneur, 1990).

- Valéry, Paul, Lettre d'un ami, Monsieur Teste, Paris (1896), Gallimard coll. «L'Imaginaire », 1999.

- Verne, Jules, Michel Strogoff (1876), Paris, Le livre de poche, nº 2034, 1994. 